\title{
Simultaneous occlusion of three cilioretinal arteries following scleral buckling surgery under local anesthesia
}

This article was published in the following Dove Press journal:

International Medical Case Reports Journal

14 September 2016

Number of times this article has been viewed

\section{Pietro Emanuele Napoli* \\ Alberto Cuccu* \\ Roberta Farci \\ Maurizio Fossarello}

Department of Surgical Sciences, Eye Clinic, University of Cagliari, Cagliari, Italy

*These authors contributed equally to this work
Correspondence: Pietro Emanuele Napoli Department of Surgical Sciences, Eye Clinic, University of Cagliari, via Ospedale 46, 09124 Cagliari, Italy

Email pietronapoli@ymail.com
Background: Cilioretinal artery (CRA) occlusions are rare in young patients. In these cases, the most commonly associated causes are considered to be the same as those implicated in central retina artery occlusions, such as vasculitic processes, migraine, cardiac disorder, and coagulation abnormality. The aim of this article was to report for the first time the medical records and investigational results of an unusual case of simultaneous occlusion of three CRAs after scleral buckling surgery under local anesthesia.

Methods: A complete ophthalmic examination, including color fundus image, fundus fluorescein angiography, optical coherence tomography, visual field, as well as systemic and laboratory assessments, was performed.

Results: A case of contemporaneous blockage of three CRAs after ab externo surgery for retinal detachment in a 29-year-old Caucasian woman was reported. The interdisciplinary approach and the imaging results have allowed us the clinical definition of such a very rare case.

Conclusion: Here, we reported that optical coherence tomography is an indispensable tool to better delineate the pathological process and follow atrophic changes in the macula, especially in cases in which fundus fluorescein angiography and systemic tests may be poorly informative.

Keywords: cilioretinal artery occlusion, optical coherence tomography, retinal detachment

\section{Introduction}

The causes of cilioretinal artery (CRA) occlusion are considered to be the same as those implicated in central retina artery occlusion, such as coagulation abnormality, vasculitic processes, migraine, and cardiac disorder.

Clinically, the occlusion of the CRA appears as an area of retinal whitening, representing ischemic cloudy swelling of the inner layers of retina. In general, fundus fluorescein angiography (FFA) shows a delayed fluorescein filling and emptying of the irrigation area of these arteries.

Although recovery of retinal circulation is possible, the inner retinal layer usually shows atrophic changes in the late stage of evolution. In these cases, FFA performed a week or more lately may not show perfusion abnormalities.

Relatively recently, optical coherence tomography (OCT) has improved the diagnostic ability to delineate different eye diseases in both the anterior and posterior segments, including acute and longstanding retinal artery occlusions. ${ }^{1-3}$

Here we report an unusual case of occlusion of three CRAs after scleral buckling surgery under local anesthesia. 


\section{Case report}

A 29-year-old Caucasian woman presented at our Eye Clinic after a left eye accidental contusion. The best-corrected visual acuity (BCVA) was 20/20 in the right eye and light perception in the contralateral eye.

Slit lamp examination was unremarkable in the right eye, while external ocular findings and anterior segment abnormalities in the left eye included eyelid ecchymosis, a subconjunctival hemorrhage, a corneal abrasion, and a total hyphema. Intraocular pressure (IOP), as assessed by means of Goldmann applanation tonometry, was $14 \mathrm{mmHg}$ in the right eye and $15 \mathrm{mmHg}$ in the left eye.

Two days after, therapy with tranexamic acid (500 mg twice per day) was started. Consequently, hyphema disappeared, and BCVA improved to 20/20 in the left eye. A retinal

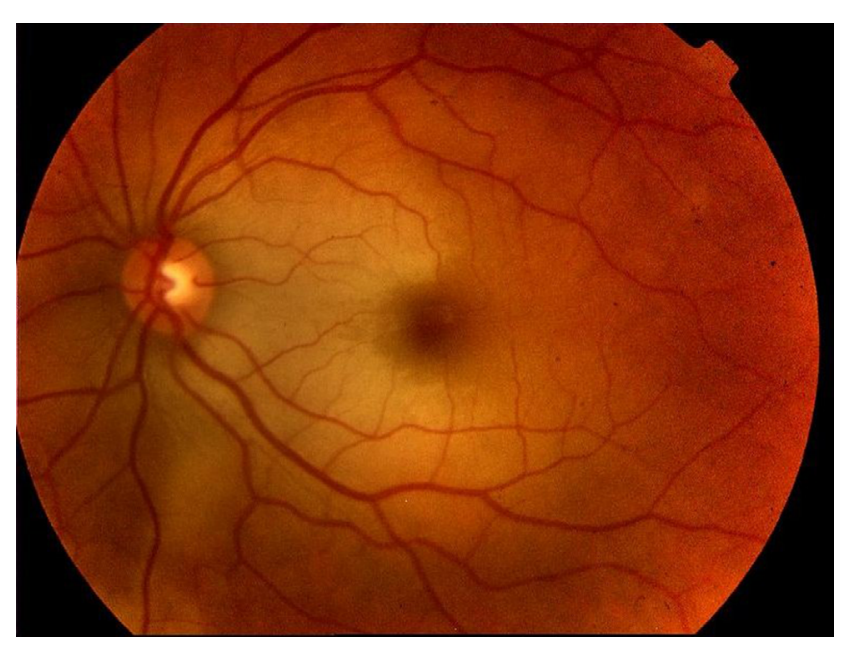

Figure I Color fundus image of the left eye showed an area of retina whitening, extending along the distribution of three cilioretinal arteries.

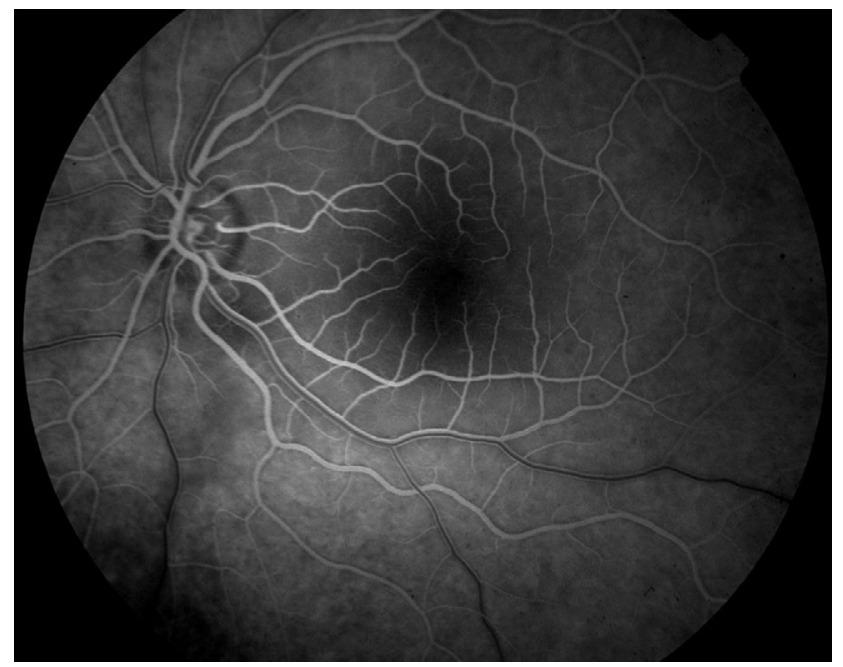

Figure 2 Fluorescein angiography images revealed only a vertical choroidal watershed centered on the optic disk, a faint screen effect in the perifoveal area due to intraretinal edema, and a normal CRA perfusion.

Abbreviation: CRA, cilioretinal artery. examination revealed a retinal dialysis and a retinal detachment in the inferotemporal sector, with macula on. Retinal cryotherapy, scleral buckling (14 $\mathrm{mm}$ behind the limbus), and evacuative puncture were performed in local anesthesia. At the end of the surgery, the retina had flattened, and the central retinal artery was normally perfused.

In the first postoperative day, the left eye visual acuity decreased again to counting finger.

Moreover, the assessment of the direct photomotor reflex revealed a poor response to light of the left pupil. The IOP was $14 \mathrm{mmHg}$. At the dilated fundus examination, a flattened retina with subtle pale elevation within the macular region extending from the temporal edge of the optic disk to the fovea was observed. All these clinical and fundus features were compatible with the simultaneous occlusion of three CRAs emerging from the temporal edge of the optic disk (Figure 1). At the following day, FFA evidenced only a vertical choroidal watershed centered on the optic disk, a faint screen effect in the perifoveal area due to intraretinal edema, and a normal CRA perfusion (Figure 2). The right eye angiography was unremarkable. An OCT (Zeiss Cirrus HDOCT; Carl Zeiss Meditec AG, Jena, Germany) examination revealed an increased reflectivity and a diffuse thickening of the inner retinal layers (Figure 3).

At visual field examination (SITA-Fast Central 30-2 strategy; Carl Zeiss Meditec AG), central scotoma and generalized retinal sensitivity reduction, with a foveal threshold of $5 \mathrm{~dB}$, mean defect of $-13.68 \mathrm{~dB}$, and pattern standard deviation of $10.35 \mathrm{~dB}$, were diagnosed (Figure 4A). Laboratory tests showed a white cell count

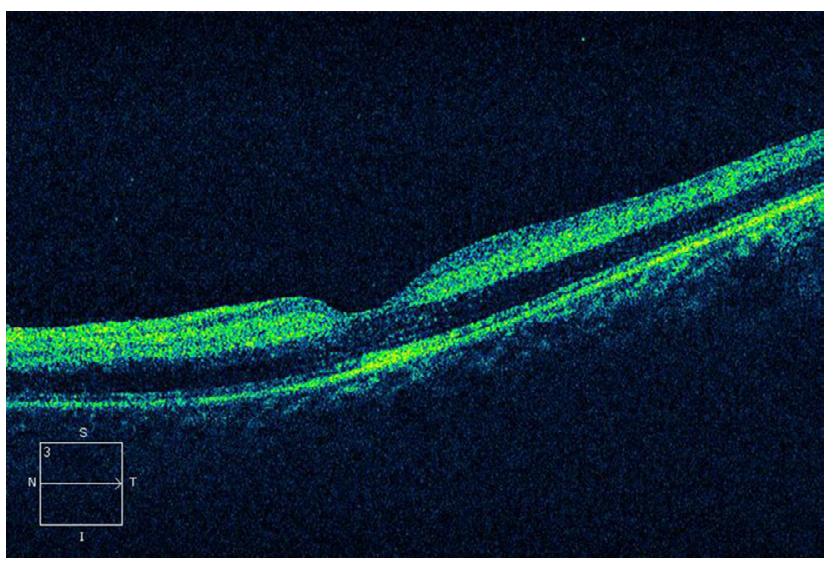

Figure 3 A horizontal, cross-sectional OCT tomogram through the fovea disclosed diffuse thickening of the neurosensory retina.

Notes: Increased reflectivity was noted in the inner retinal layers from the surface of retina to the photoreceptor layers. Decreased reflectivity was observed from the photoreceptor layers and the retinal pigment epithelium secondary to the shadowing effect. Foveolar depression, photoreceptor layer in the fovea, and retinal pigment epithelium underneath the fovea were normal.

Abbreviations: OCT, optical coherence tomography; $\mathrm{N}$, nasal; $\mathrm{S}$, superior; $\mathrm{T}$, temporal; I, inferior. 


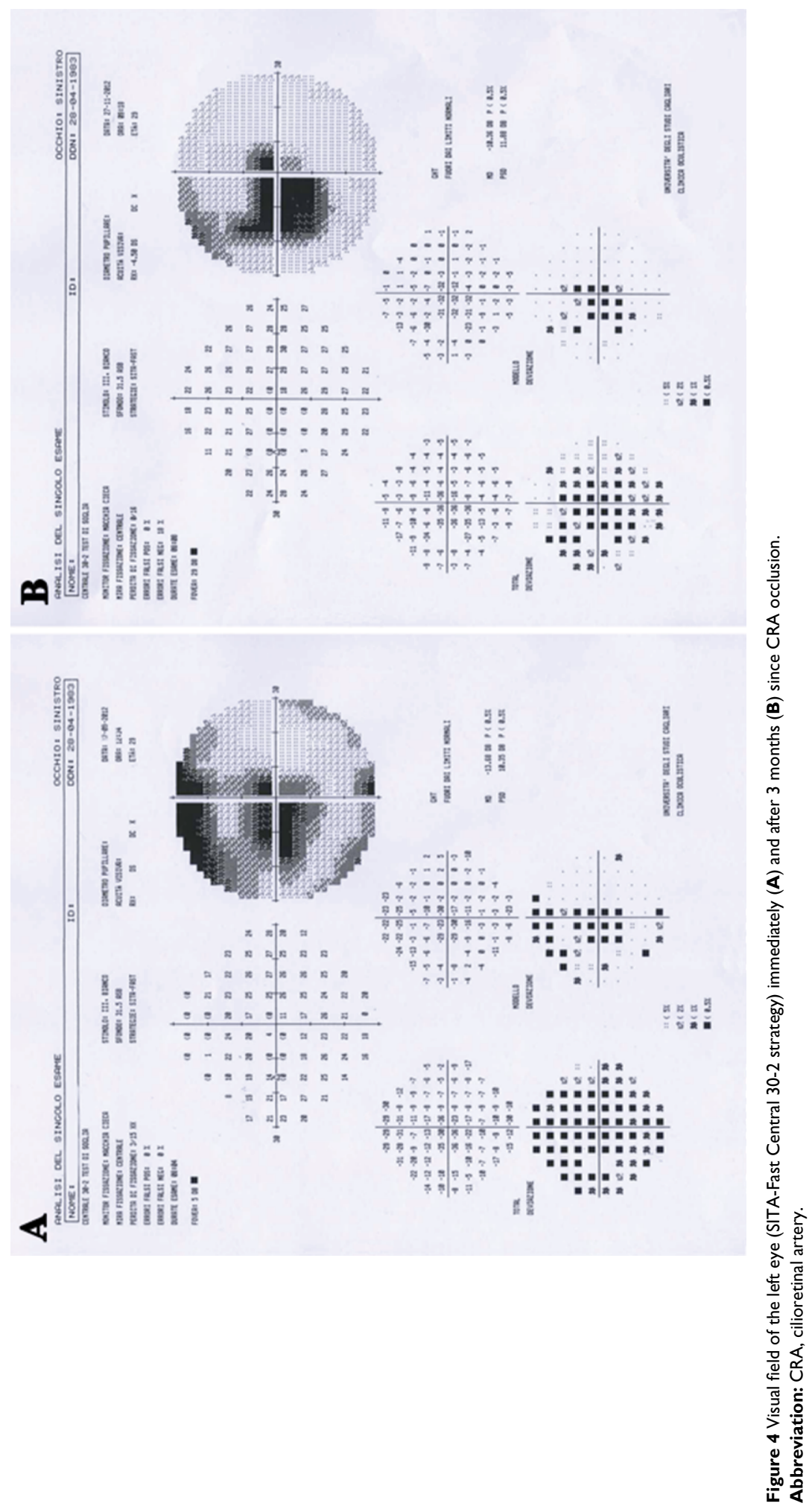




\begin{tabular}{llll} 
D: & CZM6611478 & Exam date: & 11/27/2012 \\
DOB: & $4 / 28 / 1983$ & Exam time: & 8:31 AM \\
Gender: & Female & Technician: & Operator, Cirrus \\
Doctor: & & Signal strength: & $4 / 10$ \\
\hline
\end{tabular}

\begin{tabular}{ll|l}
\hline Macula thickness: macular cube $512 \times 128$ & OD $\bigcirc$ & OS \\
\hline
\end{tabular}
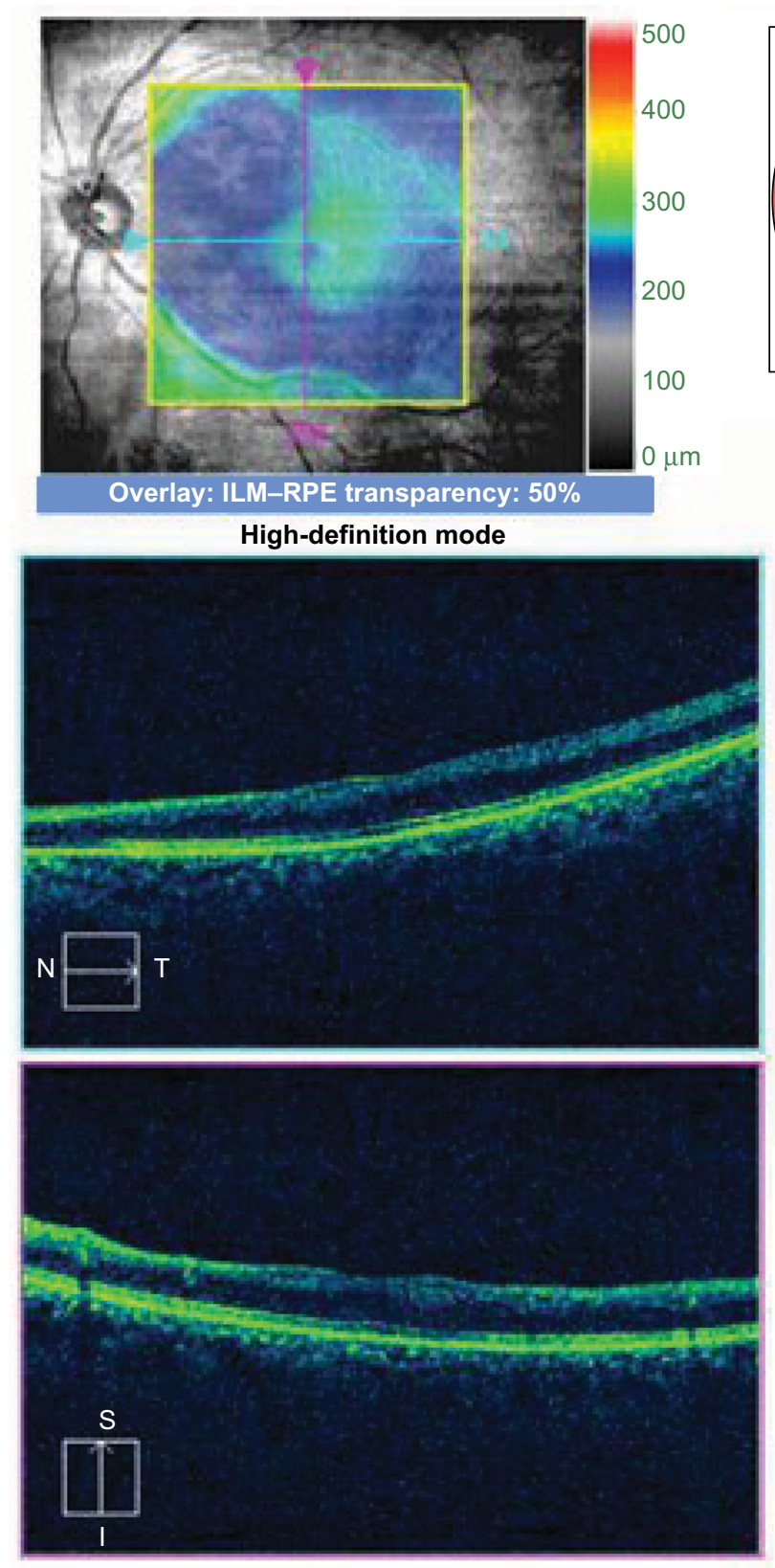

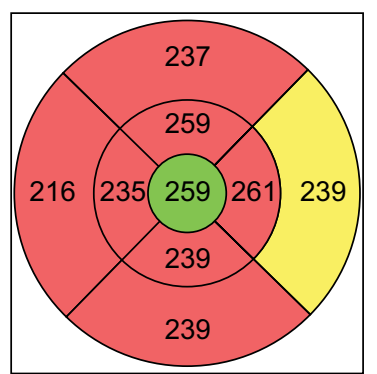

ILM-RPE thickness $(\mu \mathrm{m})$

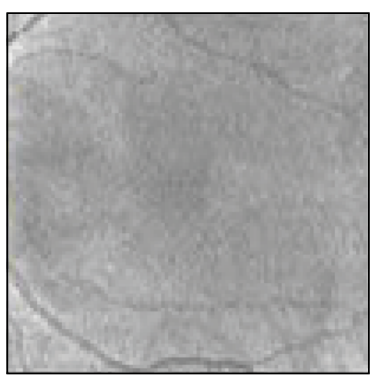

Fovea: 267.70

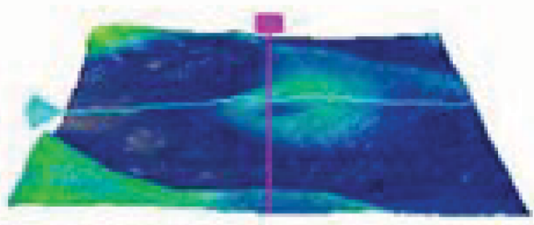

ILM-RPE

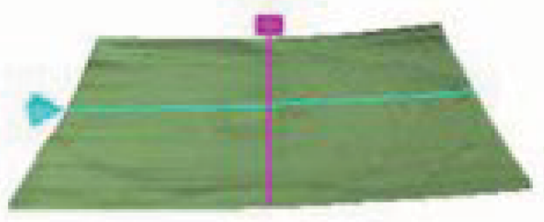

ILM

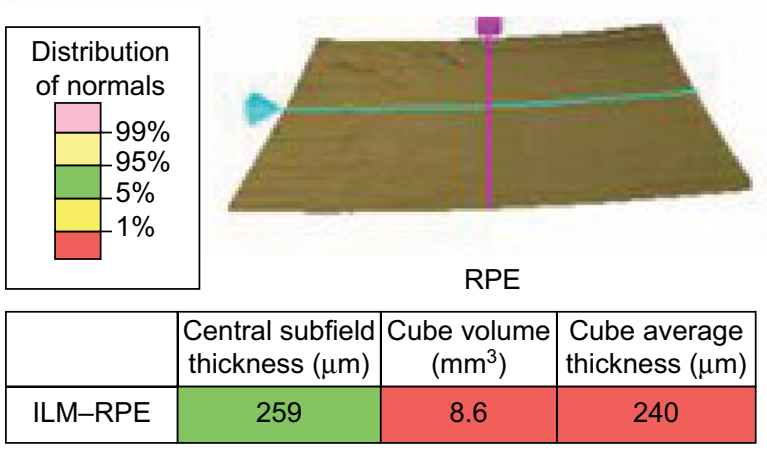

Figure 5 The macular thickness map, which was generated by means of OCT after 3 months since CRA occlusion, demonstrated thinning along the distribution of three cilioretinal arteries.

Abbreviations: OCT, optical coherence tomography; CRA, cilioretinal artery; ID, identity; DOB, date of birth; OD, right eye; OS, left eye; ILM, internal limiting membrane; RPE, retinal pigment epithelium; N, nasal; S, superior; T, temporal; I, inferior.

of $13.3 \times 10^{3} / \mu \mathrm{L}$ (normal range: $4.00-10.00 \times 10^{3} / \mu \mathrm{L}$ ), a hematocrit of $35.7 \%$ (normal range: $38.0 \%-48.0 \%$ ), and a total serum protein of $5.6 \mathrm{~g} / \mathrm{dL}$ (normal range: $6.6-8.3 \mathrm{mg} / \mathrm{dL})$. All other clinical examinations, including cardiology workup, carotid ultrasound, and immunological and coagulation tests, were negative.
Three months later, BCVA in the left eye improved to 20/20. Anterior segment and fundus examination were unremarkable. No atrophy or focal pallor of the optic disk was observed, and retinal whitening was completely resolved. FFA was unremarkable. OCT imaging evidenced macular atrophy subsequent to the acute edematous change in the eye and dif- 
fuse macular thinning (Figure 5). ${ }^{4}$ The visual field showed the reduction of central scotoma with a foveal threshold of $29 \mathrm{~dB}$, mean defect of $-10.36 \mathrm{~dB}$, and pattern standard deviation of $11.88 \mathrm{~dB}$ (Figure 4B). Written informed consent to publish this case report and the accompanying images was obtained from the patient.

\section{Discussion}

Scleral buckling is an effective surgical procedure in treating rhegmatogenous retinal detachment. Although this simple method of repair is associated with a high anatomic success rate, well-known postoperative complications, some of which are rare, may occur. However, no case of simultaneous occlusion of CRAs after scleral buckling under local anesthesia has been, to our knowledge, reported before in the literature.

As previously reported, intraoperative manipulations of the globes may cause IOPs greater than the central retinal artery perfusion pressures. Since cilioretinal arteries arise from the posterior ciliary arteries, a significant peak in IOP might result in their occlusion. However, in our case, there is no direct or indirect sign of compression of the central retinal vein due to scleral buckling or encirclement. ${ }^{5,6}$

Here, scleral buckling was performed after local anesthesia; therefore, localized vasoconstriction from the anesthetic or mechanical pressure from the bolus of the anesthetic solution may have occurred during the surgery, thus determining an obstruction of the CRA and an ischemic injury in the cilioretinal field. ${ }^{7,8}$ In fact, although the exact pathomechanism of this complication is unknown, retrobulbar injections have been associated with postoperative visual loss because of ischemia to the visual pathways. ${ }^{9}$

Also, retinal vascular occlusion after orbital locoregional anesthesia has been described after phacoemulsification, but is rare. ${ }^{10}$

Generally, in patients younger than 40 years, different clinical examinations are suggested to evaluate whether a hypercoagulable state or a vasculitic process is implied in the occlusive event. However, in this clinical case, all these tests were normal.

Furthermore, retinal neurons may be damaged after reperfusion by means of mechanisms of chemical oxidation, which may explain the late atrophy of the inner retinal layers.

With regard to the final visual restoration, in our case report, it may be explained by foveal perfusion from the temporal side by branches of superior branch retinal artery. In fact, as evidenced by the visual field at the third month, the patient revealed a normal foveal sensitivity despite a central scotoma in the temporal half (with SITA-Fast Central 30-2 strategy) and a restored inferotemporal central area corresponding to the aforementioned superior temporal artery. Moreover, quantitative
OCT at the third month detected thinning along the distribution of three cilioretinal arteries and an intact central subfield.

Ophthalmologists who examine patients with areas of retinal whitening, especially with the support of OCT, should consider paracentral acute middle maculopathy in the differential diagnosis, which is an isolated or plurifocal (as in case of central retinal vein occlusion) ischemic event of the deep capillary plexus of the retina. ${ }^{11}$ Clearly, in our case report, the area of retinal whitening is noticeably distinct, appearing more opaque and white in color (neither gray-white nor wedgeshaped), more superficial in the retina following the distribution of the nerve fiber layer (as assessed by OCT) and the CRAs, and more extensive than that potentially related to the focal areas of retinal capillary ischemia. Moreover, the dark aspect of retinal layers underneath hyperreflectivity due to the shadowing of the upper layers is absent in our case. Therefore, the aforementioned ocular findings are more compatible with occlusion of CRAs rather than paracentral acute middle maculopathy.

\section{Conclusion}

The interdisciplinary approach and the imaging results have allowed us the clinical definition of such an unusual case. Clearly, when this complication occurs, carotid disease, and local or systemic factors, should be evaluated. However, although cardiovascular investigations and laboratory workup are useful to assess potential risk factors, OCT is an indispensable tool to better delineate the pathological process and follow atrophic changes in the macula (in our report, OCT imaging after 3 months shows thinning of the inner retina in the previously thickened nasal retina, which indicates permanent damage of this area), especially in cases in which FFA and systemic tests are poorly informative.

\section{Disclosure}

None of the authors has a conflict of interest in this work. No financial support was received for this work.

\section{References}

1. Napoli PE, Coronella F, Satta GM, Galantuomo MS, Fossarello M Evaluation of the adhesive properties of the cornea by means of optical coherence tomography in patients with meibomian gland dysfunction and lacrimal tear deficiency. PLoS One. 2014;9(12):e115762.

2. Napoli PE, Coronella F, Satta GM, Fossarello M. A novel technique of contrast-enhanced optical coherence tomography imaging in evaluation of clearance of lipids in human tears. PLoS One. 2014;9(11):e109843.

3. Mathew R, Papavasileiou E, Sivaprasad S. Autofluorescence and highdefinition optical coherence tomography of retinal artery occlusions Clin Ophthalmol. 2010;4:1159-1163.

4. Chen SN, Hwang JF, Chen YT. Macular thickness measurements in central retinal artery occlusion by optical coherence tomography. Retina. 2011;31(4):730-737.

5. Schatz H, Fong AC, McDonald HR, et al. Cilioretinal artery occlusion in young adults with central retinal vein occlusion. Ophthalmology. 1991;98:594-601. 
6. Ohkubo H. Fluorescein angiographic findings in the detached and reattached retina. Jpn J Ophthalmol. 1988;32:423-428.

7. Villa SR, Méndez RS, Martín MC, García MC. Central retinal artery occlusion after phacoemulsification under peribulbar anaesthesia: pathogenic hypothesis. Arch Soc Esp Oftalmol (English Edition). 2016;91(1):40-43.

8. Feibel RM, Guyton DL. Transient central retinal artery occlusion after posterior sub-Tenon's anesthesia. J Cataract Refract Surg. 2003;29(9):1821-1824.
9. Morgan CM, Schatz H, Cantrill AK, et al. Ocular complications associated with retrobulbar injections. Ophthalmology. 1988;95(5): 660-665.

10. Stambough JL, Dolan D, Werner R, Godfrey E. Ophthalmologic complications associated with prone positioning in spine surgery. JAm Acad Orthop Surg. 2007;15(3):156-165.

11. Rahimy E, Sarraf D, Dollin ML, Pitcher JD, Ho AC. Paracentral acute middle maculopathy in nonischemic central retinal vein occlusion. $A m$ J Ophthalmol. 2014;158(2):372-380.

\section{Publish your work in this journal}

The International Medical Case Reports Journal is an international, peer-reviewed open-access journal publishing original case reports from all medical specialties. Previously unpublished medical posters are also accepted relating to any area of clinical or preclinical science. Submissions should not normally exceed 2,000 words or
4 published pages including figures, diagrams and references. The manuscript management system is completely online and includes a very quick and fair peer-review system, which is all easy to use. Visit $\mathrm{http}: / / \mathrm{www}$.dovepress.com/testimonials.php to read real quotes from published authors.

Submit your manuscript here: https://www.dovepress.com/international-medical-case-reports-journal-journal 